\title{
A German genome-wide linkage scan for type 2 diabetes supports the existence of a metabolic syndrome locus on chromosome 1p36.13 and a type 2 diabetes locus on chromosome 16p12.2
}

\author{
K. Hoffmann • M. Mattheisen • S. Dahm • \\ P. Nürnberg • C. Roe • J. Johnson • N. J. Cox • \\ H. E. Wichmann • T. F. Wienker • J. Schulze • \\ P. E. Schwarz • T. H. Lindner
}

Received: 30 September 2006 / Accepted: 4 February 2007 / Published online: 27 April 2007

(C) Springer-Verlag 2007

\begin{abstract}
Aims/hypothesis The aim was to identify type 2 diabetes susceptibility regions in 250 German families.

Subjects and methods We conducted a genome-wide linkage scan using 439 short tandem repeat polymorphisms at an average resolution of $7.76 \pm 3.80 \mathrm{cM}$ (Marshfield). In an affected-only-design (affected sib pairs), we performed nonparametric multipoint linkage analyses. Conditional analyses were applied where linkage signals were found in the baseline analyses.
\end{abstract}

Electronic supplementary material The online version of this article (doi:10.1007/s00125-007-0658-4) contains supplementary material, which is available to authorised users.

\section{K. Hoffmann}

Institute of Medical Genetics, Charité, University Medical School,

Humboldt University,

Berlin, Germany

\section{Mattheisen • T. F. Wienker}

Institute for Medical Biometry, Informatics and Epidemiology,

University of Bonn,

Bonn, Germany

S. Dahm

Department of Bioinformatics,

Max Delbrück Center for Molecular Medicine Berlin Buch,

Berlin, Germany

P. Nürnberg

Cologne Center for Genomics and Institute for Genetics,

University of Cologne,

Cologne, Germany

C. Roe $\cdot$ N. J. Cox

Department of Human Genetics, The University of Chicago,

Chicago, IL, USA
Results We identified two loci with nominal evidence for linkage on chromosomes 1p36.13 and 16p12.2 (D1S3669, $37.05 \mathrm{cM}$, logarithmic odds ratio $[\mathrm{LOD}]=1.49, p=0.004$; D16S403, $43.89 \mathrm{cM}, \mathrm{LOD}=1.85, p=0.002) . \mathrm{D} 16 \mathrm{~S} 403$ crossed the empirically obtained threshold of genome-wide suggestive significance of $L O D=1.51$. Positive findings in those regions have been reported by the following other linkage studies on: (1) symptomatic/clinical gall bladder disease with type 2 diabetes in Mexican Americans from the San Antonio Family Diabetes/Gallbladder Study (LOD=3.7, D1S1597-D1S407, 29.93-33.75 cM); (2) body

\author{
J. Johnson \\ Advocate Illinois Masonic Medical Center, \\ Chicago, IL, USA \\ H. E. Wichmann \\ Institute of Epidemiology, \\ GSF-Research Center for Environment and Health, \\ Neuherberg, Germany \\ J. Schulze $\cdot$ P. E. Schwarz \\ Department of Internal Medicine III, \\ University Clinic Carl Gustav Carus, \\ Technical University Dresden, \\ Dresden, Germany \\ T. H. Lindner $(\bowtie)$ \\ Department of Nephrology and Hypertension, \\ Community Hospital Nuremberg, Medical Clinic 4, \\ Friedrich Alexander University of Erlangen-Nuremberg, \\ Breslauer Str. 201, \\ 90471 Nürnberg, Germany \\ e-mail: t-lindner@gmx.de
}


size-adiposity in another Mexican American population (D1S1597, LOD =2.53, $29.93 \mathrm{cM}$ ); (3) lipid abnormalities (LOD=3.1, D1S2826-D1S513, 41.92-60.01 cM); and (4) hypertension in Australian sib pairs (LOD=3.1, D1S2834 D1S2728, 31.02-33.75 cM); as well as (5) a meta-analysis of four European type 2 diabetes-related genome scans $(\mathrm{LOD}=1.09, \mathrm{D} 16 \mathrm{~S} 412,42.81 \mathrm{cM})$. In linkage analyses conditional on evidence for linkage at D16S403 we identified a LOD increase $(\Delta \mathrm{LOD})$ of $1.55(p=0.0075)$ at D17S2180. Similar conditioning on D17S2180 revealed evidence for interaction with $\mathrm{D} 1 \mathrm{~S} 3669(\Delta \mathrm{LOD}=1.67, p=0.0055)$, D16S403 $(\Delta \mathrm{LOD}=1.48, p=0.0091)$ and another locus on chromosome 1 where several genome scans have reported evidence for linkage ( $200 \mathrm{cM}, \Delta \mathrm{LOD}=1.60, p=0.0066)$. Conclusions/interpretation Our results and the findings of other studies are consistent with the presence of a locus for a complex metabolic syndrome on chromosome $1 \mathrm{p} 36.13$.

Keywords Clinical science - Conditional linkage analysis . Genetics of type 2 diabetes - Genomewide scan .

Linkage studies $\cdot$ Metabolic syndrome .

Susceptibility region

\section{Abbreviations \\ LOD logarithmic odds ratio \\ Prop proportional to the evidence for linkage}

\section{Introduction}

Whereas the identification of at least six genes for the rare autosomal-dominant forms of MODY was remarkably successful, only modest progress has been made for common type 2 diabetes. The first successful genome-wide scan in 330 affected Mexican American sib pairs with type 2 diabetes found linkage to chromosome 2q37 (D2S125D2S140, logarithmic odds ratio $[\mathrm{LOD}]=4.15)$. Using an association approach, a haplo(geno)type consisting of three polymorphisms with functional impact was identified within the calpain-10 gene [1]. The study was successful because another interacting locus on chromosome 15obtained by conditional analyses - was considered [2].

In Germany, type 2 diabetes shows increasing prevalence with 5-8 million people having some form of diabetes (prevalence: 6-10\%). In an effort to identify causative genetic factors, we report here results of linkage studies in which we identified two type 2 diabetes loci. We elucidated potentially interacting regions by conditioning our sample on the positive linkage signals identified. Taken together, our results and the findings of other studies provide evidence for a complex metabolic syndrome locus on chromosome $1 \mathrm{p} 36.13$.

\section{Subjects and methods}

Subjects Patients were identified through the Department of Metabolic Disorders of the University Clinic Dresden and through diabetologists in private practice. Blood samples were used for DNA extraction and baseline laboratory parameters. Patients were all of German origin living in Saxony (south-eastern Germany). The ethics committee approved this study; informed consent was obtained from all subjects. Primary inclusion criteria were: (1) only affected subjects with type 2 diabetes according to WHO criteria; (2) at least two affected sibs per sibship; and (3) age at diagnosis $\geq 35$ years. Patients were excluded if they had: (1) suspected MODY (at least three generations with autosomal-dominant diabetes; at least 2 years of treatment with diet and/or glucose-lowering drugs other than insulin; age at diagnosis $\leq 25$ years); or (2) type 1 diabetes (atypical manifestation of type 2 diabetes, with antibody testing for islet cell antibodies, insulin, GAD64 being conducted in such cases and in 359 randomly selected patients). Parents were not available for our study. Clinical characteristics of the sample are summarised in Table 1.

Genotyping We screened 439 microsatellite markers with an average marker density of $7.76 \pm 3.80 \mathrm{cM}$ (Marshfield), no single intermarker distance of $>15 \mathrm{cM}$, an average heterozygosity of $0.765 \pm 0.068$ and a call rate of $\geq 95 \%$. Five markers in Hardy-Weinberg disequilibrium were

Table 1 Clinical characteristics of affected individuals included in the analysis

\begin{tabular}{lll}
\hline Parameter & Women & Men \\
\hline Subject number & 300 & 231 \\
Height $(\mathrm{m})$ & $1.62 \pm 0.07$ & $1.73 \pm 0.08$ \\
Weight $(\mathrm{kg})$ & $77.36 \pm 13.83$ & $83.56 \pm 14.08$ \\
BMI $\left(\mathrm{kg} / \mathrm{m}^{2}\right)$ & $29.53 \pm 4.81$ & $27.73 \pm 3.81$ \\
Blood pressure systolic/diastolic & & \\
(mmHg) & & \\
Systolic & $147 \pm 20$ & $143 \pm 18$ \\
Diastolic & $82 \pm 10$ & $82 \pm 10$ \\
Age at diagnosis (years) & $51 \pm 13$ & $47 \pm 12$ \\
Treatment $(\%)$ & & \\
Diet & 50.66 & 50.66 \\
Oral hypoglycaemic agents & 60.74 & 63.76 \\
Insulin & 45.19 & 47.16 \\
HbA 1 (c) & $7.21 \pm 1.22$ & $7.54 \pm 1.87$ \\
Creatinine $(\mu \mathrm{mol} / \mathrm{l})$ & $92.52 \pm 42.02$ & $103.41 \pm 34.77$ \\
Uric acid $(\mu \mathrm{mol} / \mathrm{l})$ & $282.29 \pm 96.37$ & $298.15 \pm 97.91$ \\
Total cholesterol (mmol/l) & $6.12 \pm 1.40$ & $6.01 \pm 2.62$ \\
Total triacylglycerol (mmol/1) & $2.53 \pm 1.96$ & $2.35 \pm 1.70$ \\
HDL-cholesterol (mmol/l) & $1.27 \pm 0.39$ & $1.18 \pm 0.47$ \\
LDL-cholesterol $(\mathrm{mmol} / \mathrm{l})$ & $3.74 \pm 1.10$ & $3.57 \pm 1.63$ \\
\hline
\end{tabular}

Total number of affected individuals: 531. Data are means \pm standard deviation (SD). 
removed (AFMA312XG5, D3S2395, D7S820, D11S2632, D14S617). Mendelian/genotyping errors were identified by Pedcheck [3] and Merlin [4], and removed or retyped, respectively.

Genetic relationships among family members were checked by Graphical Relationship Representation [5]. Misclassification of half-siblings as full siblings (19 families with 22 half-sib pairs) was identified and resolved. Four affected sib pairs with identical genotypes of both siblings (either not previously reported as twins or possible sample duplication), four identical twins and four affected sib pairs where siblings previously reported to be related turned out to be unrelated to each other were removed. We analysed 250 families comprising 290 affected sib pairs and 22 half-sib pairs and corresponding to 531 affected non-founders (231 males, 300 females). The sample structure was as follows: 203 (28) families with two (three) affected siblings, 16 families with two affected individuals in a half-sib relationship and three families with two affected siblings and one affected half-sibling.

Linkage analyses, simulations All analyses were run under the graphical user interface easyLINKAGE (http://source forge.net/project/showfiles.php?group_id=124875). Marker allele frequencies were estimated from our sample. Nonparametric two-point linkage analysis was performed with Splink [6, 7], multipoint linkage analysis with Merlin using the score-pairs statistic. Accurate estimates of the genomewide statistical significance of linkage results were obtained empirically by simulating 1,000 replicates derived from our dataset using Merlin and by performing the locus-counting approach [8]. Allegro [9] was used for conditional analyses. The evaluation of the significance of $p$ values and the generation of family weights were performed as described recently [2].

Statistical power, simulation studies For details [see Electronic supplementary material (ESM)].

\section{Results}

Two-point nonparametric linkage analyses revealed six regions on chromosomes $1,2,3,7,11$ and 16 with a $L O D \geq 1$. The strongest signal was obtained on chromosome $3(\mathrm{LOD}=2.87$, nominal $p=0.0003$ at $\mathrm{D} 3 \mathrm{~S} 3050)$ (ESM Table 1). Two of those loci reached a nonparametric multipoint $\mathrm{LOD}>1$ (D1S3669, LOD $=1.49$, nominal $p=0.004$; $\mathrm{D} 16 \mathrm{~S} 403, \mathrm{LOD}=1.85$, nominal $p=0.002$ ) (Fig. 1; ESM Table 2). The region around D16S403 crossed the level of genome-wide suggestive linkage (ESM Table 3).
Conditional analyses revealed no significant interaction between chromosomes 1 and 16 (ESM Table 4; ESM Fig. 1a,b, ESM Fig. 2a,b). However, when conditioning on D16S403, we identified an interaction with D17S2180 $\left(\mathrm{LOD}_{\text {baseline/prop }}=0.08 / 1.63, p=0.0075\right.$ for LOD increase $[\triangle \mathrm{LOD}]$, where prop=proportional to the evidence for linkage; ESM Fig. 1c,d, ESM Fig. 2b). When conditioning on D17S2180, the interval D1S3669-D1S1622 showed a $\Delta$ LOD from 1.14 to $2.81 \quad(p=0.0055$, weight $0-1)$ and a region on chromosome 1 at $\sim 200 \mathrm{cM}$ also showed a significant $\Delta \mathrm{LOD}(\Delta \mathrm{LOD}=1.60, p=0.0066$; ESM Fig. 1e,f, ESM Fig. 2c). In addition, the LOD score at D16S403 increased from 1.85 to 3.33 ( $p=0.0091$, weight prop).

\section{Discussion}

Our hypothesis is that the clinical heterogeneity of type 2 diabetes is related to underlying genetic heterogeneity. Thus we focused our studies on families with a more homogeneous form of diabetes transmission, using clinical and laboratory-based measurements to minimise contamination by other known subtypes of diabetes such as MODY or type 1 diabetes, and by recruiting patients from a defined geographical area with German ancestry.

In both the two- and multipoint linkage analyses, two loci on chromosomes 1 (37.05 cM, D1S3669) and $16(43.89 \mathrm{cM}$, D16S403) showed nominal and suggestive evidence for linkage, respectively. Close to our finding on chromosome 1 a LOD $=3.7$ was found at marker interval D1S1597-D1S407 (29.93-33.75 cM) in Mexican American patients with gall bladder disease; $46 \%$ of these patients also had type 2 diabetes. When this group was analysed without diabetic individuals, that locus disappeared, possibly indicating that components related to type 2 diabetes are important genetic or pathogenetic factors [10]. Other studies on parameters of lipid metabolism (LOD=3.1, D1S2826-D1S513, 41.92$60.01 \mathrm{cM})$ and on hypertension in Australian sib pairs (LOD=3.1, D1S2834-D1S2728, 31.02-33.75 cM) also reported linkage here $[10,11]$. In nondiabetic Mexican Americans body size-adiposity showed suggestive evidence of linkage at D1S1597 (LOD=2.53, $29.93 \mathrm{cM})$ [12]. At D1S1597 Iwasaki et al found nominal evidence for linkage $(\mathrm{LOD}=0.77, p=0.030)$ in an autosomal genome scan for type 2 diabetes genes and genes affecting BMI in Japanese people (164 families, 256 affected sib pairs) [13]. Considering our results, the results from those studies and the fact that the duration of diabetes and poor blood sugar control are strongly associated with gall bladder disease, the existence of a potential diabetes/metabolic syndrome locus seems possible.

Our locus on chromosome 16 was among the linkage signals in a genome-scan meta-analysis approach including 
Fig. 1 Nonparametric multipoint LOD plot (MERLIN) using a linear allele-sharing model. Black arrow, locus on chromosome 1; open arrow, locus on chromosome 16. Vertical grey lines below the $x$-axis show the relative positions of typed markers

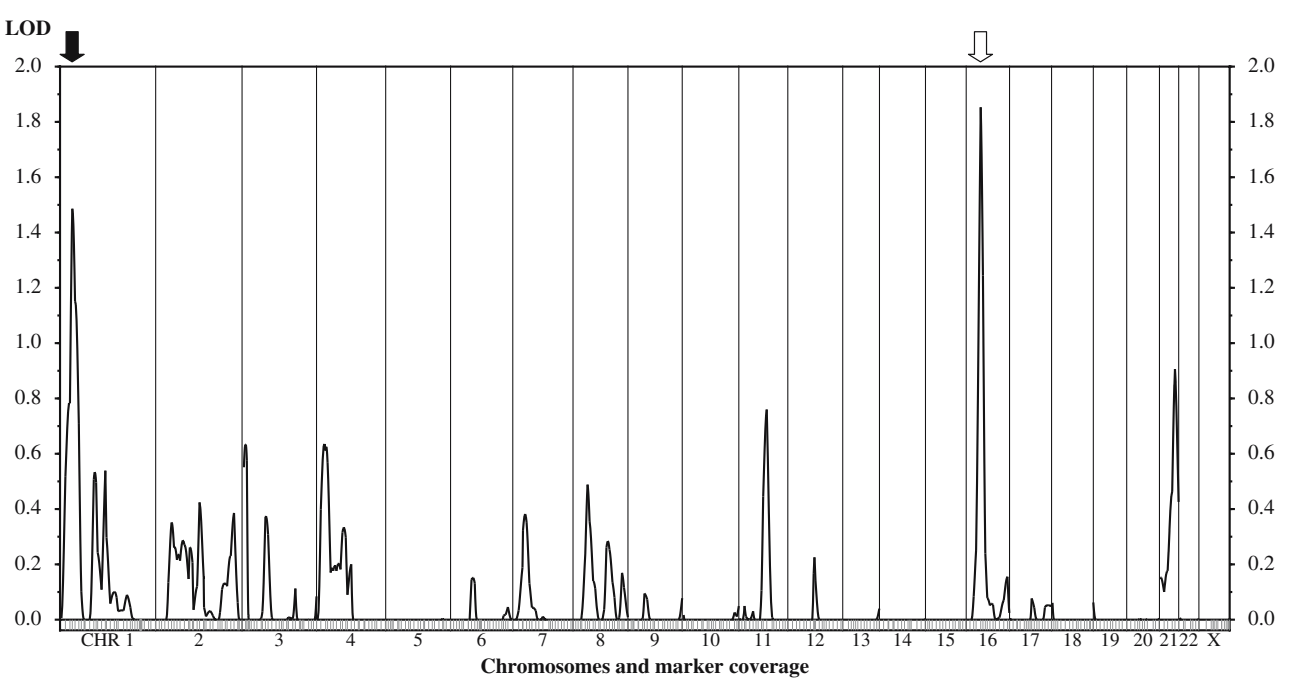

four European genome scans (Botnia I, Botnia II, British, French samples) [14]. The two Botnia samples (LOD= $0.78 / 1.41)$ and the pooled data $(\mathrm{LOD}=1.09)$, but not the British and French samples mapped type 2 diabetes to our region (among other loci).

Neither of the two loci on chromosomes 1 and 16 reached genome-wide significance; however, D16S403 crossed the empirically obtained threshold of "suggestive" significance of $\mathrm{LOD}=1.51$, while $\mathrm{D} 1 \mathrm{~S} 3669(\mathrm{LOD}=1.49)$ was very close to that level (ESM Table 3). According to our simulations, the levels for suggestive/significant evidence for linkage shifted from $\mathrm{LOD}=2.20 / 3.63$ [15] to $\mathrm{LOD}=1.51 / 2.74$, due to the difference between the actual amount of information extracted from our genome screen (which was conducted without parental information and using an infinitely dense screen with perfectly informative markers).

We tested locus interactions by performing conditional linkage analyses as described recently [2]. Here, we found no evidence for interaction between the identified loci on chromosomes 1 and 16. However, the conditioning on D16S403 revealed an interaction with the region around D17S2180 (ESM Table 4). The strongest findings from a meta-analysis of four European genome scans were close to this region; this meta-analysis also highlighted the region on chromosome 16 that we also identified, as noted above [14]. Finally, conditioning on D17S2180 led to a $\Delta$ LOD of our findings at D1S3669 and D16S403 which indicated interaction between those loci. Interestingly, another broad region on chromosome $1(170-200 \mathrm{cM})$ that was highlighted in these interaction analyses is one in which several other genome scans reported evidence for linkage [16-19].

Our study was the first to investigate German type 2 diabetic patients in a genome-wide linkage scan. However, diabetes as a subphenotype was studied in a German sample with myocardial infarction or coronary revascular- isation procedure (diabetes in $16.3 \%$ of 618 affected sib pairs with myocardial infarction, diabetes in $8.4 \%$ of 238 unaffected sibs) revealing an $\mathrm{LOD}=2.96$ at chromosome 6 (D6S1277, $173.31 \mathrm{cM}$ ) [20]. In our sample no positive linkage signal was seen in this region, possibly due to the different sample composition with respect to myocardial infarction and population substructure. Several studies have reported a locus around the MODY1 region on chromosome 20 [21-24]. In our sample we were unable to replicate either those findings or the region with recent association results around this locus. Also, we found no evidence for linkage on chromosome $2 \mathrm{q} 37$, suggesting that the heavily studied calpain-10 region does not appear to play a major role in the pathogenesis of type 2 diabetes in Germans. Similarly, we found no evidence for linkage to the region around DG10S478, where compelling associations with type 2 diabetes in Icelandic, Danish and US cohorts have been reported [25].

Acknowledgements We thank all our patients from the Saxony region for participating in this study. In particular, we thank the many Saxonian general practitioners for contributing affected sib pairs to our study. K. Hoffmann was supported by the Deutsche Forschungsgemeinschaft (DFG [German Research Foundation], SFB 577, project A4) and is a recipient of a Rahel Hirsch Fellowship, provided by the Medical Faculty of the Berlin Charité Hospital. This study was funded as part of the DFGForschergruppe (Research Group) (DFG FOR 423) "Genetic epidemiology and medical genetics of complex diseases" (project C1: Molecular genetics of type 2 diabetes in Germany [T. H. Lindner, H. E. Wichmann, T. F. Wienker, M. Mattheisen]). Our work was also supported by the German Federal Ministry of Science and Education through the National Genome Research Network (P. Nürnberg). T. H. Lindner was supported by grants from the DFG (LiDFG768/3-1/3-3, LiDFG768/4-1/4-2/6-1).

Duality of interest The authors have no commercial relationships or duality of interest relevant to this manuscript. 


\section{References}

1. Horikawa Y, Oda N, Cox NJ et al (2000) Genetic variation in the gene encoding calpain-10 is associated with type 2 diabetes mellitus. Nat Genet 26:163-175

2. Cox NJ, Frigge M, Nicolae DL et al (1999) Loci on chromosomes 2 (NIDDM1) and 15 interact to increase susceptibility to diabetes in Mexican Americans. Nat Genet 21:213-215

3. O'Connell JR, Weeks DE (1998) PedCheck: a program for identification of genotype incompatibilities in linkage analysis. Am J Hum Genet 63:259-266

4. Abecasis GR, Cherny SS, Cookson WO, Cardon LR (2002) Merlin - rapid analysis of dense genetic maps using sparse gene flow trees. Nat Genet 30:97-101

5. Abecasis GR, Cherny SS, Cookson WO, Cardon LR (2001) GRR: graphical representation of relationship errors. Bioinformatics 17:742-743

6. Holmans P (1993) Asymptotic properties of affected-sib-pair linkage analysis. Am J Hum Genet 52:362-374

7. Holmans P, Clayton D (1995) Efficiency of typing unaffected relatives in an affected-sib-pair linkage study with single-locus and multiple tightly linked markers. Am J Hum Genet 57:1221-1232

8. Wiltshire S, Cardon LR, McCarthy MI (2002) Evaluating the results of genomewide linkage scans of complex traits by locus counting. Am J Hum Genet 71:1175-1182

9. Gudbjartsson DF, Jonasson K, Frigge ML, Kong A (2000) Allegro, a new computer program for multipoint linkage analysis. Nat Genet 25:12-13

10. Puppala S, Dodd GD, Fowler S et al (2006) A genomewide search finds major susceptibility loci for gallbladder disease on chromosome 1 in Mexican Americans. Am J Hum Genet 78:377-392

11. Glenn CL, Wang WY, Benjafield AV, Morris BJ (2000) Linkage and association of tumor necrosis factor receptor 2 locus with hypertension, hypercholesterolemia and plasma shed receptor. Hum Mol Genet 9:1943-1949

12. Cai G, Cole SA, Freeland-Graves JH, MacCluer JW, Blangero J, Comuzzie AG (2004) Principal component for metabolic syndrome risk maps to chromosome $4 p$ in Mexican Americans: the San Antonio Family Heart Study. Hum Biol 76:651-665

13. Iwasaki N, Cox NJ, Wang YQ et al (2003) Mapping genes influencing type 2 diabetes risk and BMI in Japanese subjects. Diabetes 52:209-213

14. Demenais F, Kanninen T, Lindgren CM et al (2003) A metaanalysis of four European genome screens (GIFT Consortium) shows evidence for a novel region on chromosome 17p11.2-q22 linked to type 2 diabetes. Hum Mol Genet 12:1865-1873

15. Lander E, Kruglyak L (1995) Genetic dissection of complex traits: guidelines for interpreting and reporting linkage results. Nat Genet $11: 241-247$

16. Hanson RL, Ehm MG, Pettitt DJ et al (1998) An autosomal genomic scan for loci linked to type II diabetes mellitus and bodymass index in Pima Indians. Am J Hum Genet 63:1130-1138

17. Vionnet N, Hani El H, Dupont S et al (2000) Genomewide search for type 2 diabetes-susceptibility genes in French whites: evidence for a novel susceptibility locus for early-onset diabetes on chromosome 3q27-qter and independent replication of a type 2diabetes locus on chromosome 1q21-q24. Am J Hum Genet 67:1470-1480

18. Wiltshire S, Hattersley AT, Hitman GA et al (2001) A genomewide scan for loci predisposing to type 2 diabetes in a U.K. population (the Diabetes UK Warren 2 Repository): analysis of 573 pedigrees provides independent replication of a susceptibility locus on chromosome 1q. Am J Hum Genet 69:553-569

19. Ng MC, So WY, Cox NJ et al (2004) Genome-wide scan for type 2 diabetes loci in Hong Kong Chinese and confirmation of a susceptibility locus on chromosome 1q21-q25. Diabetes 53:1609-1613

20. Broeckel U, Hengstenberg C, Mayer B et al (2002) A comprehensive linkage analysis for myocardial infarction and its related risk factors. Nat Genet 30:210-214

21. Bowden DW, Sale M, Howard TD et al (1997) Linkage of genetic markers on human chromosomes 20 and 12 to NIDDM in Caucasian sib pairs with a history of diabetic nephropathy. Diabetes 46:882-886

22. Love-Gregory LD, Wasson J, Ma J et al (2004) A common polymorphism in the upstream promoter region of the hepatocyte nuclear factor-4 alpha gene on chromosome 20q is associated with type 2 diabetes and appears to contribute to the evidence for linkage in an Ashkenazi Jewish population. Diabetes 53:1134-1140

23. Zouali H, Hani EH, Philippi A et al (1997) A susceptibility locus for early-onset non-insulin dependent (type 2) diabetes mellitus maps to chromosome $20 \mathrm{q}$, proximal to the phosphoenolpyruvate carboxykinase gene. Hum Mol Genet 6:1401-1408

24. Permutt MA, Wasson JC, Suarez BK et al (2001) A genome scan for type 2 diabetes susceptibility loci in a genetically isolated population. Diabetes 50:681-685

25. Grant SF, Thorleifsson G, Reynisdottir I et al (2006) Variant of transcription factor 7-like 2 (TCF7L2) gene confers risk of type 2 diabetes. Nat Genet 38:320-323 